\title{
Population balance modelling for fertilizer granulation process
}

\author{
Ludmila Vesjolaja $^{1} \quad$ Bjørn Glemmestad ${ }^{2} \quad$ Bernt Lie $^{1}$ \\ ${ }^{1}$ Department of Electrical Engineering, IT and Cybernetics, University of South-Eastern Norway, Norway, \\ \{ludmila.vesjolaja, bernt. lie\} @usn. no \\ ${ }^{2}$ Process Modeling and Control Department, Yara Technology Center, Norway, bjorn.glemmestadeyara.com
}

\begin{abstract}
Few granulation plants are operated optimally. It is common to operate granulation plants below their maximum design capacity, and in many cases, periodic instabilities may also occur. From a process control and optimization point of view, it is desirable to develop a dynamic model that can show the dominating dynamics of a granulation process and can be used for design of optimal operation of the granulation plant. In this paper, a dynamic model of a drum granulator is developed using population balance (PB). Different simulation scenarios are used to analyze various granulation mechanisms that are characteristic to drum granulators. Simulation results show that for the drum granulator, the particle agglomeration has a greater impact on the change in particle size distribution (PSD) compared to the particle growth due to layering. In addition, coarser particles are produced when a sizedependent agglomeration kernel is used in the granulator model. For combined processes, i.e., processes where the particle growth due to layering and agglomeration are considered simultaneously, coarser particles with a wider PSD are obtained with the size-dependent agglomeration kernel.
\end{abstract}

Keywords: granulation, population balance, agglomeration, layering

\section{Introduction}

Granulation is a particle enlargement process during which fine particles and/or atomizable liquids are converted into granules via a series of complex physical processes. In a typical granulation plant, the main operational goal is to produce granules with improved properties compared to their ungranulated form, and therefore, to meet product quality requirements (e.g., produce granules with the desired PSD, moisture content, porosity, etc.). Granulation processes are used in a wide range of industrial applications, such as in pharmaceuticals, chemicals, and fertilizer industries (Litster and Ennis, 2004). However, the operation of granulation plants in an industrial scale can be challenging. Many granulation plants have a high recycle-to-product ratio, and it is common to operate granulation plants below their maximum design capacity. In addition, periodic instability associated with the operation of the granulation circuit have been reported (Radichkov et al., 2006; Heinrich et al., 2003). This causes the PSD of the particles flowing out of the granulator to oscillate, thus making it difficult to maintain the desired product quality. An increase in the production of off-spec particles (oversized and undersized) gives rise to a higher recycleto-product ratio, and the plant does not operate in an optimal manner. One way to address these problems is to develop a mathematical model of the granulator that can be used to study and understand various dynamics occurring in the granulator. The model can further be used to design optimal control structures to increase the efficiency of the plant.

The most widely used approaches for modeling granulation processes include Discrete Element Modeling, as well as PB modeling. In this study, since the focus is on the development of a dynamic model suitable for control purposes, PB modeling has been used to develop a mathematical model of a drum granulator. A rich literature related to PB modeling of a granulation process is available (Randolph and Larson, 1962; Wang et al., 2006; Wang and Cameron, 2007). In these works, (i) the effect of different granulation mechanisms to the PSD of the granulator outflow is neglected, (ii) the numerical scheme (Hounslow discretization method) used for solving the population balance equation (PBE) is relatively inaccurate (shows overprediction as also mentioned in (Kumar, 2006; Kumar et al., 2006)), and (iii) many of the processes are only batch processes. In this paper, a dynamic model of a continuous drum granulator using the PB framework is developed. The resulting integro-differential PBEs are discretized using an accurate numerical scheme, namely the cell average technique (Kumar, 2006) and the flux limitation scheme (Koren, 1993). The developed model is simulated to understand and analyze how different granulation mechanisms affect the PSD of the granules formed in the granulator.

\section{Granulation Mechanisms}

According to (Iveson et al., 2001), the granulation process is divided into three basic mechanisms: (i) nucleation and wetting, (ii) growth and consolidation, and (iii) breakage and attrition. Different granulation mechanisms are predominant depending on the type of the granulator being used. For continuous drum granulation with recycling, effects of nucleation, breakage, and attrition mechanisms are believed to be negligible compared to particle growth due to layering and agglomeration (Fig. 1). Particle nu- 


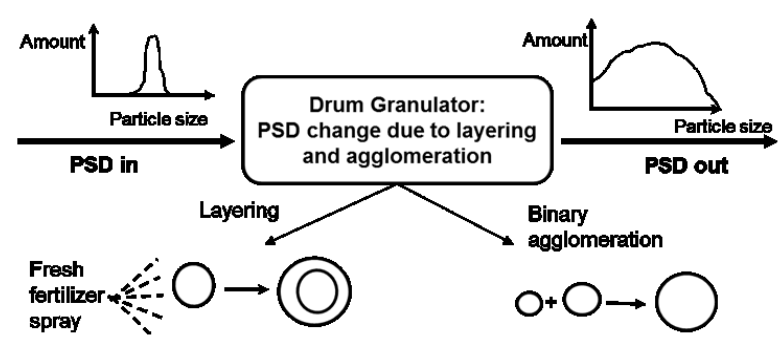

Figure 1. Main granulation mechanisms characteristic to drum granulators.

cleation is insignificant for this particular process as it is a continuous process in which the recycle feed acts as seeds for the granulator. Particle breakage is mainly important in a high shear granulators, e.g., granulation mechanism due to breakage can be significant in pharmaceutical industries where high shear granulators are typically used. As to the attrition, this granulation mechanism might give significant changes in PSD only when high velocities (e.g., fluidized bed spray) granulators are used (Litster and Ennis, 2004). Layering occurs due to a successive coating of a liquid phase onto a granule. As a result, the granule grows in its mass, and the volume increases, but the number of granules in the system remains unchanged. No collision between granules is assumed during this particle growth (Litster and Ennis, 2004). Layering is a continuous process (differential growth), and an assumption of sizeindependent linear growth rate is common in the PB modeling of granulation processes. This simplification implies that each granule has the same exposure to a new fertilizer spray feed material, and a volumetric growth rate is proportional to a projected granule surface area (Litster and Ennis, 2004). Binary agglomeration refers to a particle growth mechanism that occurs due to successful collision of two particles, resulting in the formation of a larger composite particle. Agglomeration is a discrete (sudden) process that changes the total number of particles: two particles die, and a new particle is born as a result of collision of two particles. Thus, the agglomeration results in a reduction in the total number of particles, while the total mass remains conserved (Litster and Ennis, 2004).

\section{Model Development}

\subsection{Population Balance Principles}

Balance laws such as mass and energy balances are often used in process modeling to describe dynamics of different physical and chemical processes. With particulate processes, PB is frequently used to describe dynamics of particle property distribution. A detailed derivation and explanation of the PBE can be found in Ramkrishna (Ramkrishna, 2000). The general form of a PBE with particle diameter $(L)$ as the internal coordinate is represented as,

$$
\begin{aligned}
\frac{\partial n(L, t)}{\partial t}=-\frac{\partial}{\partial L}[G n(L, t)]+ & B(L, t)-D(L, t) \\
& -\frac{\partial}{\partial z}\left[\frac{d z}{d t} n(L, z, t)\right],
\end{aligned}
$$

where $n(L, t)$ is the number density function. The first term on the right hand side represents the particle growth due to layering, the second and the third terms stand for particle birth and death respectively, and the last term represents a continuous process and gives the flow of particles through the granulator. $G$ is the growth rate and $z$ represents the distance along the axial direction of the drum granulator. The birth and the death terms usually include integrals which make the solution of the population balance equation complicated. In this paper, a plug flow along the axial direction of the drum granulator has been assumed. For simplifying the model complexity, a concept of output equivalent (perfect mixing) inside the granulator can be assumed. Thus, Eq. (1) can be simplified to

$$
\begin{aligned}
\frac{\partial n(L, t)}{\partial t}=-\frac{\partial}{\partial L}[G n(L, t)]+B(L, t) & -D(L, t) \\
& +\dot{n}_{\mathrm{i}} \gamma_{\mathrm{i}}-\dot{n}_{\mathrm{e}} \gamma_{\mathrm{e}}
\end{aligned}
$$

Here, $\dot{n}_{\mathrm{i}}$ is the number flowrate of particles entering the granulator (influent), $\dot{n}_{\mathrm{e}}$ is the number flowrate of particles leaving the granulator (efluent), $\gamma_{i}$ is the size distribution function of the inlet flow of the particles (influent), $\gamma_{\mathrm{e}}$ is the size distribution function of the outlet flow of the particles (efluent).

In addition, for the PBE of Eq. (2), the following assumptions are made:

- The concept of perfect mixing inside the granulator is applied: particle property (size) inside the granulator is the same as at the outlet of the granulator.

- Particle breakage in neglected since the drum granulator is operating at low shear forces. Thus, the birth $(B)$ and death $(D)$ rates are only due to binary agglomeration.

- Particle size reduction due to attrition is neglected since the granulation drum does not operate at high velocities.

\subsection{Growth Rate for Layering}

The formulation of the particle growth rate for layering $(G)$ is based on combination of the work of (Mörl, 1981) and (Mörl et al., 1977), as summarized in (Drechsler et al., 2005). This model assumes linear size-independent growth rate, meaning that a small particle gets less slurry per unit time than a larger particle, but the growth rate (the change of particle diameter over time) is constant for all particle sizes. Thus, the growth rate due to layering depends on a slurry rate (fresh fertilizer spray rate, $\dot{m}_{\mathrm{sl}}$ ), 
moisture fraction in the slurry $\left(X_{\mathrm{sl}, \mathrm{i}}\right)$, and the total surface area of the particles $\left(A_{\mathrm{p}, \text { tot }}\right)$ as given by Eqs. (3) and (4).

$$
\begin{gathered}
G=\frac{\partial L}{\partial t}=\frac{2 \dot{m}_{\mathrm{sl}}\left(1-X_{\mathrm{sl} \mathrm{i}}\right)}{\rho A_{\mathrm{p}, \mathrm{tot}}} \\
A_{\mathrm{p}, \mathrm{tot}}=\pi n \int_{L=0}^{L=\infty} L^{2} d L .
\end{gathered}
$$

\subsection{Particle Agglomeration}

Particle agglomeration is a discrete event, which is challenging to model. One of the most widely used formulations of the agglomeration process was introduced by (Kapur and Fuerstenau, 1969). The general form of a lengthbased agglomeration is represented by Eqs. (5) and (6),

$$
\begin{aligned}
& B(L, t)=\frac{L^{2}}{2} \\
& \times \int_{0}^{L} \frac{\beta\left[\left(L^{3}-\lambda^{3}\right)^{\frac{1}{3}}, \lambda\right] n\left[\left(L^{3}-\lambda^{3}\right)^{\frac{1}{3}}, t\right] n(\lambda, t)}{\left(L^{3}-\lambda^{3}\right)^{\frac{2}{3}}} d \lambda,
\end{aligned}
$$

$$
D(L, t)=n(L, t) \int_{0}^{\infty} \beta(L, \lambda) n(\lambda, t) d \lambda .
$$

Here, $\beta$ is the agglomeration (coalescence) kernel. The agglomeration kernel is a key parameter that controls the overall rate of agglomeration. Despite more than 50 years of research, only empirical and semi-empirical agglomeration kernels are available. Thus, these should be fitted to experimental data. Some of the most frequently used agglomeration kernels for two colliding particles with volumes $v$ and $w$ in granulation processes are summarized in Table 1 . Here, $\beta_{0}$ is the part of the agglomeration kernel which usually depends on the operating conditions of the granulator such as the drum speed, bed depth and the moisture content in the particles. In this paper, the value of $\beta_{0}$ has been taken to be a constant (however tunable) for simplifying the model development. For a more detailed analysis, $\beta_{0}$ should be expressed as a function of process parameters and fitted with the experimental data. This has been left as a potential future work.

Table 1. Overview of agglomeration kernels.

\begin{tabular}{ll}
\hline Agglomeration kernel & References \\
\hline$\beta=\beta_{0}$ & Random kernel \\
$\beta=\beta_{0} \times(v+w)$ & Golovin (Golovin, 1963) \\
$\beta=\beta_{0} \times\left(\frac{(v+w)^{a}}{(v w)^{b}}\right)$ & Kapur (Kapur, 1972) \\
\hline
\end{tabular}

The PDE represented by Eq. (7) can be discretized into a system of ODEs using a finite volume scheme. In this paper, a high resolution scheme, based on the flux limiting approach, is chosen as the numerical scheme for particle size discretization. Particularly, the Koren flux limiting method (Koren, 1993) is used in this paper. The high resolution flux limiting methods attain higher accuracy than the first order upwind scheme. In addition, these methods also avoid spurious oscillations by applying a high order flux in the smooth regions and a low order flux near 
discontinuities. Equation (7) can be discretized with the Koren scheme as

$$
\frac{d N_{i}}{d t}=G n\left(t, L_{i-\frac{1}{2}}\right)-G n\left(t, L_{i+\frac{1}{2}}\right)+\dot{N}_{\mathrm{i}} \gamma_{\mathrm{i}}-\dot{N}_{\mathrm{e}} \gamma_{\mathrm{e}},
$$

where,

$$
n\left(t, L_{i-\frac{1}{2}}\right) \approx \frac{1}{\Delta L}\left[N_{i-1}+\frac{1}{2} \phi\left(\widetilde{\theta}_{i-\frac{1}{2}}\right)\left(N_{i-1}-N_{i-2}\right)\right]
$$

$$
n\left(t, L_{i+\frac{1}{2}}\right) \approx \frac{1}{\Delta L}\left[N_{i}+\frac{1}{2} \phi\left(\widetilde{\theta}_{i+\frac{1}{2}}\right)\left(N_{i}-N_{i-1}\right)\right] .
$$

Here, $\phi$ is the limiter function defined as

$$
\phi(\widetilde{\theta})=\max \left[0, \min \left(2 \widetilde{\theta}, \min \left(\frac{1}{3}+\frac{2 \widetilde{\theta}}{3}, 2\right)\right)\right] .
$$

Parameter $\widetilde{\theta}$ is defined as

$$
\widetilde{\theta}_{i-\frac{1}{2}}=\frac{N_{i}-N_{i-1}+\varepsilon}{N_{i-1}-N_{i-2}+\varepsilon}, \quad \widetilde{\theta}_{i+\frac{1}{2}}=\frac{N_{i+1}-N_{i}+\varepsilon}{N_{i}-N_{i-1}+\varepsilon},
$$

with a very small constant $\varepsilon$ to avoid division by zero.

In an industrial application, it is relatively easier to work with mass-based population balance equations (PBEs) instead of number-based PBEs due to: (i) PSD in a real plant is typically measured by sieving and weighting, and (ii) mass-based PBE is more convenient to use from a numerical point of view (huge number of particles compared to their masses). To convert the number-based formulation given by Eqs. (8)-(12) to a mass-based formulation, Eq. (13) is applied,

$$
N_{i}=\frac{6 M_{i}}{\pi \rho L_{i}^{3}} .
$$

Equation (13) was derived assuming that all particles are ideal spheres with constant density. After rearranging, the growth due to layering in a mass-based PBE can be represented as

$$
\begin{aligned}
\frac{d M_{i}}{d t}=L_{i}^{3}\left[G m\left(t, L_{i-\frac{1}{2}}\right)-G m(\right. & \left.\left.t, L_{i+\frac{1}{2}}\right)\right] \\
& +\dot{M}_{\mathrm{i}} \gamma_{\mathrm{i}}-\dot{M}_{\mathrm{e}} \gamma_{\mathrm{e}},
\end{aligned}
$$

where,

$$
\begin{aligned}
m\left(t, L_{i-\frac{1}{2}}\right) \approx & \frac{1}{\Delta L}\left\{\frac{M_{i-1}}{L_{i-1}}+\frac{1}{2} \phi\left(\theta_{i-\frac{1}{2}}\right)\right. \\
& \left.\times\left(\frac{M_{i-1}}{L_{i-1}^{3}}-\frac{M_{i-2}}{L_{i-2}^{3}}\right)\right\}, \\
m\left(t, L_{i+\frac{1}{2}}\right) \approx & \frac{1}{\Delta L}\left\{\frac{M_{i}}{L_{i}}+\frac{1}{2} \phi\left(\theta_{i+\frac{1}{2}}\right)\right. \\
& \left.\times\left(\frac{M_{i}}{L_{i}^{3}}-\frac{M_{i-1}}{L_{i-1}^{3}}\right)\right\},
\end{aligned}
$$

with

$$
\theta_{i-\frac{1}{2}}=\frac{\frac{M_{i}}{L_{i}^{3}}-\frac{M_{i-1}}{L_{i-1}^{3}}+\varepsilon}{\frac{M_{i-1}}{L_{i-1}^{3}}-\frac{M_{i-2}}{L_{i-2}^{3}}+\varepsilon}, \theta_{i+\frac{1}{2}}=\frac{\frac{M_{i+1}}{L_{i+1}^{3}}-\frac{M_{i}}{L_{i}^{3}}+\varepsilon}{\frac{M_{i}}{L_{i}^{3}}-\frac{M_{i-1}}{L_{i-1}^{3}}+\varepsilon}
$$

Here $M_{i}$ is the total mass of the particle in the $i^{\text {th }}$ class. The growth rate $G$ is considered to be size-independent as described in more detail in Section 3.2. The growth rate due to layering is modeled using Eqs. (3) and (4). In addition, if $T_{\mathrm{R}}$ is the retention time, then, $\dot{M}_{\mathrm{e}} \gamma_{\mathrm{e}}=\frac{M_{i}}{T_{\mathrm{R}}}$.

\subsection{Pure agglomeration}

If the change in the particle size is considered to be due to agglomeration only, Eq. (2) reduces to

$$
\frac{\partial n(L, t)}{\partial t}=B(L, t)-D(L, t)+\dot{n}_{\mathrm{i}} \gamma_{\mathrm{i}}-\dot{n}_{\mathrm{e}} \gamma_{\mathrm{e}}
$$

Analytical solutions of the pure agglomeration problems can be found in some simplified cases. Thus, numerical techniques are needed to solve the resulted PBEs. However, the discretization of agglomeration terms $(B, D)$ is more challenging compared to the growth due to layering. Agglomeration is a discrete event and the birth and death of particles can be considered to be source and sink terms, respectively. A suitable numerical scheme that is simple to implement and produce exact numerical results of some selected moments is the cell averaging technique (Kumar, 2006; Kumar et al., 2006). The cell average scheme is referred to as a sectional method, and assigns all the newborn particles within a cell more precisely compared to other sectional methods. Using the cell average scheme, Eq. (18) can be discretized with respect to the particle size as

$$
\begin{array}{r}
\frac{d N_{i}}{d t}=B_{i-1} \lambda_{i}^{-}\left(\bar{L}_{i-1}\right) H\left(\bar{L}_{i-1}-L_{i-1}\right) \\
+B_{i} \lambda_{i}^{-}\left(\bar{L}_{i}\right) H\left(L_{i}-\bar{L}_{i}\right)+B_{i} \lambda_{i}^{+}\left(\bar{L}_{i}\right) H\left(\bar{L}_{i}-L_{i}\right) \\
+B_{i+1} \lambda_{i}^{+}\left(\bar{L}_{i+1}\right) H\left(L_{i+1}-\bar{L}_{i+1}\right) \\
\quad-N_{i} \sum_{k=1}^{N_{c}} \beta_{i k} N_{k}+\dot{N}_{\mathrm{i}} \gamma_{i}-\dot{N}_{\mathrm{e}} \gamma_{\mathrm{e}} .
\end{array}
$$

Here, $N_{c}$ is the total number of particle size classes or cells. $B_{i}$ is the birth of particles in the $i^{t h}$ cell due to binary agglomeration of two particles from the $j^{t h}$ and $k^{t h}$ cell respectively, and can be expressed as

$$
B_{i}=\frac{1}{2} \sum_{j=1}^{i} \sum_{k=1}^{i} \beta_{j k} N_{j} N_{k}
$$

where condition $L_{i-\frac{1}{2}} \leq\left(L_{j}^{3}+L_{k}^{3}\right)^{\frac{1}{3}} \leq L_{i+\frac{1}{2}}$ should be fulfilled. $\beta_{j k}$ is the agglomeration kernel for binary agglomeration of particles from the $j^{\text {th }}$ and the $k^{\text {th }}$ cells. $\bar{L}_{i}$ is the 
average diameter of all the new-born particles in the $i^{t h}$ cell, and is given as

$$
\bar{L}_{i}=\left[\frac{\sum_{j=1}^{i} \sum_{k=1}^{i} \beta_{j k} N_{j} N_{k}\left(L_{j}^{3}+L_{k}^{3}\right)}{\sum_{j=1}^{i} \sum_{k=1}^{i} \beta_{j k} N_{j} N_{k}}\right]^{\frac{1}{3}},
$$

with dimensionless term $\lambda_{i}^{ \pm}(L)$ given as

$$
\lambda_{i}^{ \pm}(L)=\frac{L^{3}-L_{i \pm 1}^{3}}{L_{i}^{3}-L_{i \pm 1}^{3}}
$$

The Heaviside step function $H$ is defined as

$$
H(\widetilde{L})= \begin{cases}1, & \text { if } \widetilde{L}>0 \\ \frac{1}{2}, & \text { if } \widetilde{L}=0 \\ 0, & \text { if } \widetilde{L}<0 .\end{cases}
$$

The cell average technique can be used to preserve any two moments. Here, we have chosen to preserve the zeroth moment (total number of particles conserved) and the third moment (total mass conserved) taking the diameterbased formulation. Using Eq. (13), the mass based form of the PBE can be written as

$$
\begin{array}{r}
\frac{d M_{i}}{d t}=L_{i}^{3}\left[B_{i-1} \lambda_{i}^{-}\left(\bar{L}_{i-1}\right) H\left(\bar{L}_{i-1}-L_{i-1}\right)\right. \\
+B_{i} \lambda_{i}^{-}\left(\bar{L}_{i}\right) H\left(L_{i}-\bar{L}_{i}\right)+B_{i} \lambda_{i}^{+}\left(\bar{L}_{i}\right) H\left(\bar{L}_{i}-L_{i}\right) \\
\left.+B_{i+1} \lambda_{i}^{+}\left(\bar{L}_{i+1}\right) H\left(L_{i+1}-\bar{L}_{i+1}\right)\right]-M_{i} \sum_{k=1}^{N_{c}} \beta_{i k} \frac{M_{k}}{L_{k}^{3}} \\
+\dot{M}_{\mathrm{i}} \gamma_{i}-\dot{M}_{\mathrm{e}} \gamma_{\mathrm{e}}
\end{array}
$$

where the birth of the particles $B_{i}$ are given as

$$
B_{i}=\frac{1}{2} \sum_{j=1}^{i} \sum_{k=1}^{i} \beta_{j k} \frac{M_{j}}{L_{j}^{3}} \frac{M_{k}}{L_{k}^{3}},
$$

and the average diameter of all the new-born particles in the $i^{\text {th }}$ class is

$$
\bar{L}_{i}=\left[\frac{\sum_{j=1}^{i} \sum_{k=1}^{i} \beta_{j k} \frac{M_{j}}{L_{j}^{3}} \frac{M_{k}}{L_{k}^{3}}\left(L_{j}^{3}+L_{k}^{3}\right)}{\sum_{j=1}^{i} \sum_{k=1}^{i} \beta_{j k} \frac{M_{j}}{L_{j}^{3}} \frac{M_{k}}{L_{k}^{3}}}\right]^{\frac{1}{3}} .
$$

With $T_{\mathrm{R}}$ being the retention time, $\dot{M}_{\mathrm{e}} \gamma_{\mathrm{e}}=\frac{M_{i}}{T_{\mathrm{R}}}$.

In this paper, agglomeration kernels $\left(\beta_{j k}\right.$ and $\left.\beta_{i k}\right)$ are defined using the Kapur model (Kapur, 1972) with $a=\frac{1}{3}$ and $b=0$; this is one of the most widely used kernels for drum granulation. With the diameter-based formulation, the agglomeration kernels are given as

$$
\beta_{x y}=\left(\frac{6}{\pi}\right)^{\frac{2}{3}} \frac{1}{\rho} \beta_{0} K_{x y}=\left(\frac{6}{\pi}\right)^{\frac{2}{3}} \frac{1}{\rho} \beta_{0}\left(L_{x}^{3}+L_{y}^{3}\right)^{\frac{1}{3}} .
$$

The term $\left(\frac{6}{\pi}\right)^{\frac{2}{3}} \frac{1}{\rho}$ arises during the conversion from the number-based formulation to the mass-based formulation of PBEs. Subscript $x y$ means either $j k$ or $i k . \quad \beta_{0}$ is the particle size independent part of the agglomeration kernel. $K_{j k}$ and $K_{i k}$ are the parts of the agglomeration kernel which are particle size dependent as shown in Eq. (27).

\subsection{Combined Process}

In the case of the combined process, a change in the particle size is a result of both particle growth due to layering, and particle binary agglomeration. The number-based PBE for the combined process is represented by (2). For conversion to the mass-based PBE, (13) is used. Size discretization for the growth term $(G)$ is performed using the Koren flux limiting scheme as discussed in Section 4.1. Particle birth $(B)$ and death $(D)$ terms are size discretized using the cell averaging technique as was discussed in detail in Section 4.2. The resulting size discretized massbased PBE for the combined process is written as

$$
\begin{aligned}
& \quad \frac{d M_{i}}{d t}=L^{3}\left[G m\left(t, L_{i-\frac{1}{2}}\right)-G m\left(t, L_{i+\frac{1}{2}}\right)\right] \\
& +L_{i}^{3}\left[B_{i-1} \lambda_{i}^{-}\left(\bar{L}_{i-1}\right) H\left(\bar{L}_{i-1}-L_{i-1}\right)+B_{i} \lambda_{i}^{-}\left(\bar{L}_{i}\right) H\left(L_{i}-\bar{L}_{i}\right)\right. \\
& \left.+B_{i} \lambda_{i}^{+}\left(\bar{L}_{i}\right) H\left(\bar{L}_{i}-L_{i}\right)+B_{i+1} \lambda_{i}^{+}\left(\bar{L}_{i+1}\right) H\left(L_{i+1}-\bar{L}_{i+1}\right)\right] \\
& -M_{i} \sum_{k=1}^{N_{c}} \beta_{i k} \frac{M_{k}}{L_{k}^{3}}+\dot{M}_{\mathrm{i}} \gamma_{\mathrm{i}}-\dot{M}_{\mathrm{e}} \gamma_{\mathrm{e}}, \quad(28)
\end{aligned}
$$

where all symbols in (28) are described in previous Sections 4.1 and 4.2.

\section{Simulation Results and Discussion}

\subsection{Simulation Setup}

The discretized PBEs for a continuous drum granulation process described by Eqs. (14), (24), and (28) are solved using a $4^{\text {th }}$ order Runge-Kutta method with fixed time step. Dynamic simulations are performed using MATLAB (MATLAB, 2017). Simulations for continuous drum granulation are performed using parameters summarized in Table 2 .

Table 2. Simulation setup parameters.

\begin{tabular}{ll}
\hline Parameter & Value \\
\hline Range of L $[\mathrm{mm}]$ & $0-8$ \\
Number of classes & 80 \\
$\rho\left[\mathrm{kg} \cdot \mathrm{m}^{-3}\right]$ & 1300 \\
$\beta_{0}\left[\mathrm{~s}^{-1}\right]$ & $8.5 \cdot 10^{-11}$ \\
$T_{\mathrm{R}}[\mathrm{s}]$ & 360 \\
$\dot{m}_{\mathrm{sl}, \mathrm{i}}\left[\mathrm{kg} \cdot \mathrm{s}^{-1}\right]$ & 250 \\
$X_{\mathrm{sl}, \mathrm{i}}$ & 0.1 \\
Time step for RK4 $[\mathrm{s}]$ & 10 \\
Simulation time $[\mathrm{h}]$ & 2.5 \\
\hline
\end{tabular}




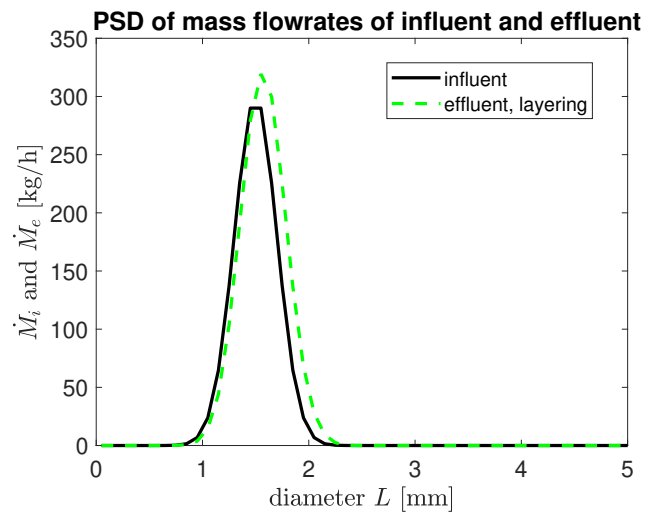

Figure 3. Influent and effluent PSD of the drum granulator for pure layering.

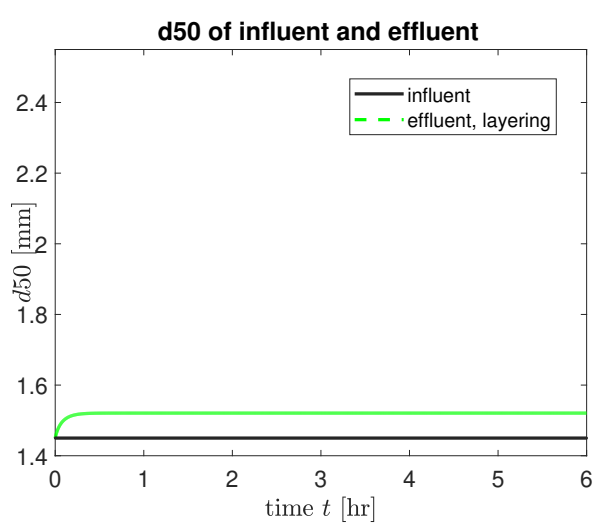

Figure 4. Change of the average particle size for pure layering.

\subsection{Simulation Results for Pure Layering and Pure Agglomeration}

In this paper, simulations results are compared by analyzing the PSD at the inlet (Gaussian distribution) and the outlet of the drum granulator. In addition, the evolution of the average size of the particles represented by their $d_{50}$ diameter (median diameter that corresponds to intercept for $50 \%$ of cumulative mass) are also studied. Figure 3 compares the PSD of the inlet flow and the outlet flow from the granulator after the system has reached the steady state. The only granulation mechanisms affecting the PSD is layering. Clearly, the PSD at the outlet of the drum granulator has changed and has become slightly wider compared to the inlet distribution. The fraction of coarser particles increases due to layering, and, thus more of large particles are produced. Figure 4 shows that the average size of the particles has increased from $1.45 \mathrm{~mm}$ to $1.52 \mathrm{~mm}$ when only the layering is the driving mechanism for particle size change. Thus, in average, particles have grown by $\sim 5 \%$ using the model parameters summarized in Table 2.

However, when agglomeration was chosen as a granulation mechanism, the average particle size has grown by $\sim 30 \%$ compared to its initial value (Figure 5 ). This increase in particle size ( $d_{50}$ to $\left.1.85 \mathrm{~mm}\right)$ was observed

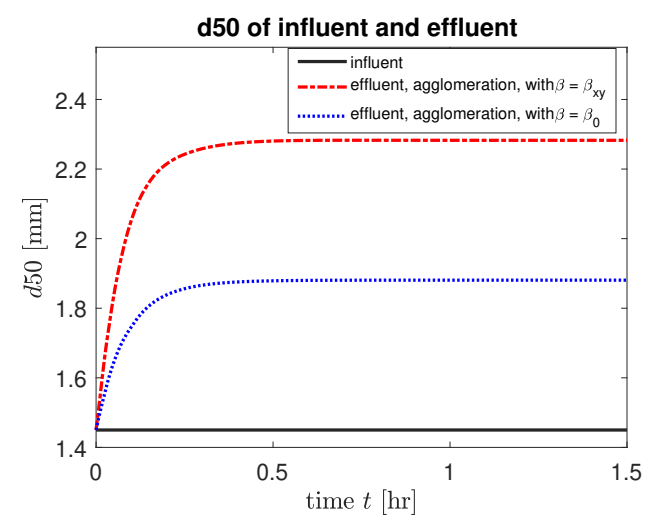

Figure 5. Change of the average particle size for pure agglomeration.

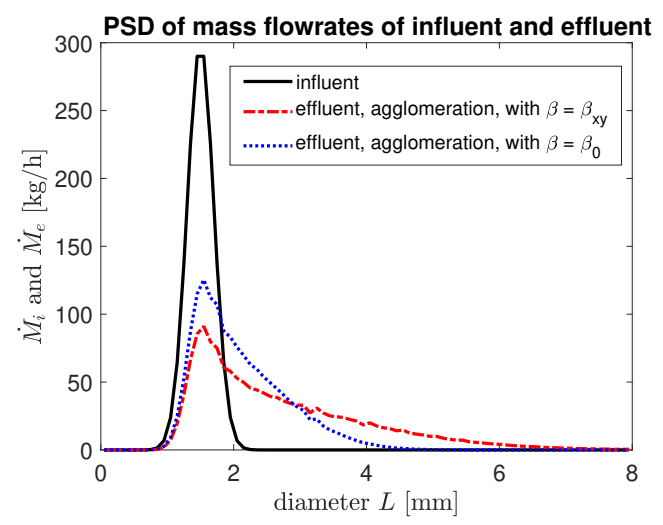

Figure 6. Influent and effluent PSD of the drum granulator for pure agglomeration.

when agglomeration was modeled using a constant (sizeindependent) agglomeration kernel. The inclusion of particle size-dependency on the agglomeration rate has increased the average particle size even more (Figure 5). The $d_{50}$ has grown from $1.45 \mathrm{~mm}$ (at the inlet) to 2.28 $\mathrm{mm}$ at the outlet of the granulator (with size-dependent agglomeration kernel). This gives $\sim 58 \%$ increase in the average particle size. As expected, the same trend is observed in PSDs of the inlet and outlet mass flow rates (Figure 6).

Granulation produces larger particles when the agglomeration rate is assumed to be dependent on particle size compared to size-independent agglomeration rate. As shown in Figure 6, agglomeration with the size-dependent kernel has produced particles whose size are as large as 5 $\mathrm{mm}$, while no particles with this size are produced with the size-independent kernel.

\subsection{Simulations Results for Combined Process}

To simulate the combined process, simultaneous particle binary agglomeration and particle growth due to layering is considered to be taking place in the drum granulator. In Figure 7, a comparison of the PSDs between pure agglomeration (with constant agglomeration kernel) and combined process is shown. 


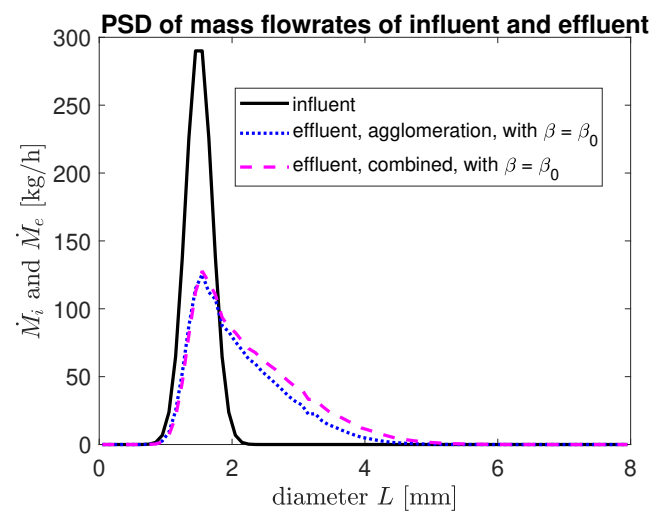

Figure 7. PSDs for pure agglomeration and combined process with size-independent kernel.

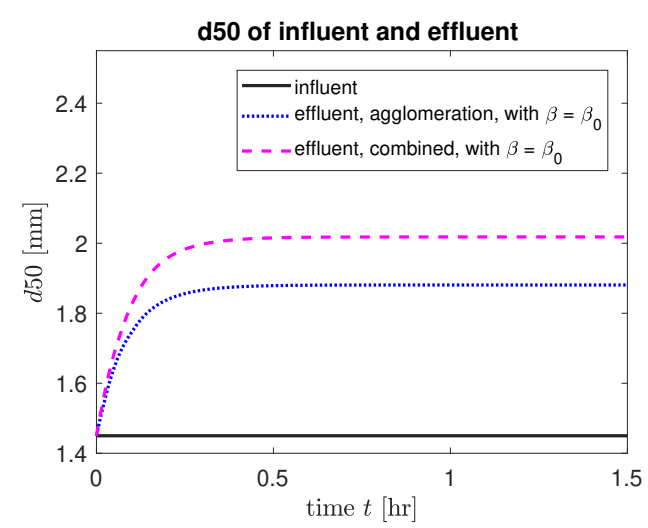

Figure 8. Change in $d_{50}$ for pure agglomeration and combined process with size-independent kernel.

For the combined process, the PSD at the outlet is wider compared to the pure agglomeration granulation process. The mass fractions of coarser particles $(>2 \mathrm{~mm})$ become larger, and hence, larger particles are produced with the combined process. The comparison of these two granulation processes with the $d_{50}$ plot (Figure 8) confirms the PSD shown in Figure 7. With the combined process, a higher value of $d_{50}$ is obtained as compared to the pure agglomeration.

The $d_{50}$ of the particles has increased by $\sim 7 \%$ (with a constant agglomeration kernel) for the combined process compared to the pure agglomeration process. A similar trend of the particle size change is observed for processes when a size-dependent agglomeration kernel is used in the simulations (Figures 9 and 10). The PSD is wider, and larger particles are produced when the combined process is simulated (Figure 9). The latter is also reflected in the $d_{50}$ plots (Figure 10). Interestingly, the value of $d_{50}$ has grown from $2.28 \mathrm{~mm}$ for the pure agglomeration process to $2.52 \mathrm{~mm}$ for the combined process (Figure 10). This gives $\sim 10 \%$ difference in average particle size for the pure and the combined process compared with the sizedependent kernel. This difference is $\sim 7 \%$ when simulations are performed with the constant agglomeration kernel. Thus, particle enlargement and hence the total change

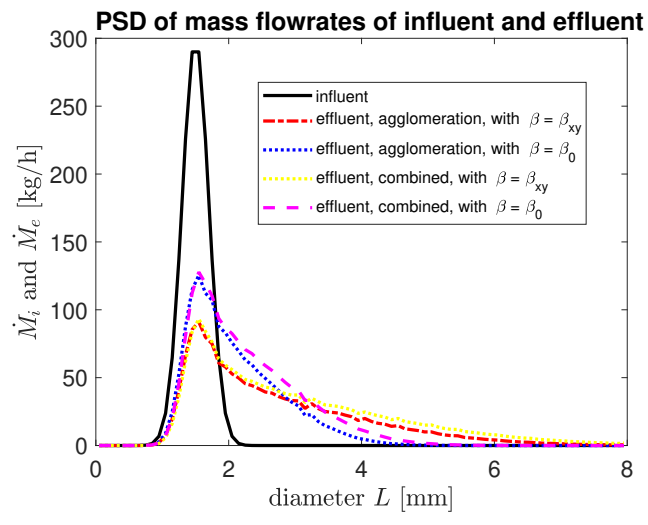

Figure 9. Comparison of PSD for pure agglomeration and combined process.

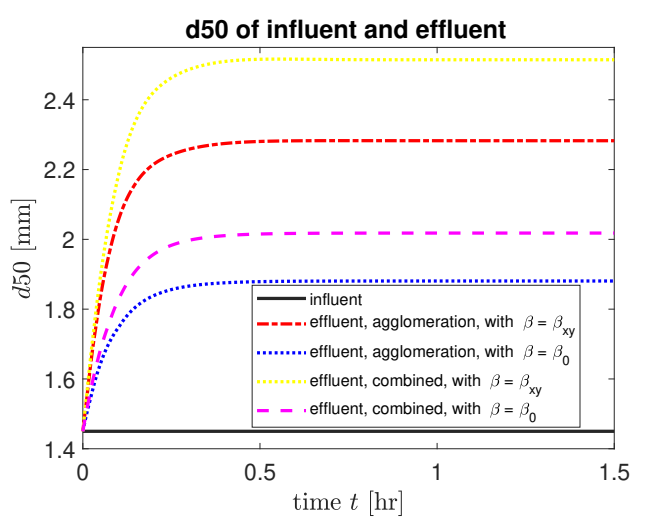

Figure 10. Comparison of $d_{50}$ for pure agglomeration and combined process.

in PSD for the combined process is more intensive if particle agglomeration is driven by a size-dependent agglomeration kernel.

The plots of PSDs (Figure 9) reveal the same pattern (larger particles are produced with the combined processes compared to pure agglomeration). The PSDs of different processes start to deviate from each other when particle size fractions are larger than $1.2 \mathrm{~mm}$. Granulation processes that are simulated with the constant agglomeration kernel produce more particles that are in the range of $[1.2,3.2] \mathrm{mm}$ of size, compared to those processes that are simulated with the size-dependent agglomeration kernel. In contrast, the processes that are simulated by assuming size-dependent agglomeration kernels, result in a larger amount of coarse particles $(\geq 3.2 \mathrm{~mm})$, e.g., simulations with the size-dependent kernel produces particles with sizes as high as $6 \mathrm{~mm}$, while no particles with such size is produced when the size-independent kernel is used (true for both pure agglomeration and combined process).

Based on the simulation results discussed above, the particle growth in drum granulators due to layering seems to play a minor role compared to the granulation mechanism for the particle binary agglomeration. This trend was indicated in others works (Wang et al., 2006; Wang and Cameron, 2007). 
The agglomeration kernel is indeed an important parameter for modeling drum granulation processes. Not only the proper formulation of the size-independent part is needed, but also the dependency of agglomeration rate to particle size should be analyzed in order to obtain a proper model of the real plant.

\section{Conclusions}

In this paper, a comparative study on various model forms for representing a drum granulation process is given. Different granulation mechanisms are compared based on simulation results represented by particle size distributions and the $d_{50}$ diameter (to reflect the average size of particles) at the influent and the effluent of the drum granulator. For the drum granulator under consideration, the simulation results lead to the following conclusions:

- Particle growth due layering has very small effect on the change of the particle sizes compared to particle binary agglomeration.

- Inclusion of the particle size dependency on the agglomeration kernel affects the mass distribution function, i.e., particles with a wider PSD and larger particles are produced compared to simulations with a constant agglomeration kernel.

- The combined process increases the growth of particles by $\sim 7 \%$ (with size-independent kernel) and by $\sim 10 \%$ (with size-dependent kernel) compared to a pure agglomeration process.

The choice of the agglomeration kernel directly affects the PSD of the particles. The size-independent part of the kernel should be calculated by taking into account the operational parameters of the actual drum granulator.

\section{Acknowledgment}

The economic support from The Research Council of Norway and Yara Technology Centre through project no. 269507/O20 'Exploiting multi-scale simulation and control in developing next generation high efficiency fertilizer technologies (HEFTY)' is gratefully acknowledged.

\section{References}

J. Drechsler, M. Peglow, S. Heinrich, M. Ihlow, and L. Mörl. Investigating the dynamic behaviour of fluidized bed spray granulation processes applying numerical simulation tools. Chemical Engineering Science, 60(14):3817-3833, 2005.

A.M. Golovin. The solution of the coagulation equation for raindrops, taking condensation into account. Soviet PhysicsDocklady, 8(2):191-193, 1963.

S. Heinrich, M. Peglow, M. Ihlow, and L. Mörl. Particle population modeling in fluidized bed-spary granulaiton - analysis of the steady state and unsteady behavior. Powder Technology, 130:154-161, 2003. doi:10.1016/S0032-5910(02)00259-0.
S.M. Iveson, J.D. Litster, K. Hapgood, and B.J. Ennis. Nucleation, growth and breakage phenomena in agitated wet granulation processes: a review. Powder technology, 117(1-2): 3-39, 2001.

PC Kapur. Kinetics of granulation by non-random coalescence mechanism. Chemical Engineering Science, 27(10):18631869, 1972.

P.C. Kapur and D.W. Fuerstenau. Coalescence model for granulation. Industrial \& Engineering Chemistry Process Design and Development, 8(1):56-62, 1969.

B. Koren. A robust upwind discretization method for advection, diffusion and source terms. In C. B. Vreugdenhil and B. Koren, editors, Numerical Methods for AdvectionDiffusion Problems, Notes on Numerical Fluid Mechanics, pages 117-138. 1993.

J. Kumar. Numerical approximations of population balance equations in particulate systems. $\mathrm{PhD}$ thesis, Otto-von-Guericke-Universität Magdeburg, Universitätsbibliothek, 2006.

J. Kumar, M. Peglow, G. Warnecke, S. Heinrich, and L. Mörl. Improved accuracy and convergence of discretized population balance for aggregation: The cell average technique. Chemical Engineering Science, 61(10):3327-3342, 2006.

J. Litster and B. Ennis. The science and engineering of granulation processes, volume 15. Springer Science \& Business Media, 2004.

MATLAB. 2017a. The MathWorks, Inc., Natick, Massachusetts, United States., 2017.

L. Mörl. Anwendungsmöglichkeiten und Berechnung von Wirbelschichtgranulationstrocknungsanlagen. $\mathrm{PhD}$ thesis, Technische Hochschule Magdeburg, 1981.

L. Mörl, M. Mittelstrab, and J. Sachse. Zum kugelwachstum bei der wirbelschichtrocknung von suspensionen oder losungen. Chemical Technology, 29(10):540-541, 1977.

R. Radichkov, T. Müller, A. Kienle, S. Heinrich, M. Peglow, and L. Mörl. A numerical bifurcation analysis of continuous fluidized bed spray granulator with external product classification. Chemical Engineering and Processing, 45:826-837, 2006. doi:10.1016/j.cep.2006.02.003.

D. Ramkrishna. Population balances: Theory and applications to particulate systems in engineering. Academic press, 2000.

A.D. Randolph and M.A. Larson. Transient and steady state size distributions in continuous mixed suspension crystallizers. AIChE Journal, 8(5):639-645, 1962.

F.Y. Wang and I.T. Cameron. A multi-form modelling approach to the dynamics and control of drum granulation processes. Powder Technology, 179(1-2):2-11, 2007.

F.Y. Wang, X.Y. Ge, N. Balliu, and I.T. Cameron. Optimal control and operation of drum granulation processes. Chemical Engineering Science, 61(1):257-267, 2006. 